\title{
ERGODICITY AND PERTURBATION BOUNDS FOR INHOMOGENEOUS BIRTH AND DEATH PROCESSES WITH ADDITIONAL TRANSITIONS FROM AND TO THE ORIGIN
}

\author{
AlEXANDER ZEIFMAN ${ }^{a, b, d}$, ANNA KOROTYSHEVA ${ }^{a, d}$, YACOV SATIN $^{a, d}, \operatorname{ViCTOR~KOROLEV~}^{c, d}$, \\ SERGEY SHORGIN $^{d}$, ROSTISLAV RAZUMCHIK ${ }^{d, e, *}$ \\ ${ }^{a}$ Department of Applied Mathematics \\ Vologda State University, S. Orlova, 6, Vologda, Russia \\ e-mail: \{a_zeifman, yacovi, a_korotysheva\}@mail.ru \\ ${ }^{b}$ Institute of Socio-Economic Development of Territories \\ Russian Academy of Sciences, Gorkogo Str., 56A, Vologda, Russia \\ ${ }^{c}$ Faculty of Computational Mathematics and Cybernetics \\ Lomonosov Moscow State University, Leninskie Gory, Moscow, Russia \\ e-mail: victoryukorolev@yandex.ru \\ ${ }^{d}$ Institute of Informatics Problems, FRC CSC \\ Russian Academy of Sciences, Vavilova str., 44-2, Moscow, 119333, Russia \\ e-mail: \{sshorgin, rrazumchik\}@ipiran.ru \\ ${ }^{e}$ Department of Applied Informatics and Probability Theory \\ Peoples' Friendship University of Russia, Miklukho-Maklaya str., 6, Moscow, 117198, Russia
}

\begin{abstract}
Service life of many real-life systems cannot be considered infinite, and thus the systems will be eventually stopped or will break down. Some of them may be re-launched after possible maintenance under likely new initial conditions. In such systems, which are often modelled by birth and death processes, the assumption of stationarity may be too strong and performance characteristics obtained under this assumption may not make much sense. In such circumstances, timedependent analysis is more meaningful. In this paper, transient analysis of one class of Markov processes defined on non-negative integers, specifically, inhomogeneous birth and death processes allowing special transitions from and to the origin, is carried out. Whenever the process is at the origin, transition can occur to any state, not necessarily a neighbouring one. Being in any other state, besides ordinary transitions to neighbouring states, a transition to the origin can occur. All possible transition intensities are assumed to be non-random functions of time and may depend (except for transition to the origin) on the process state. To the best of our knowledge, first ergodicity and perturbation bounds for this class of processes are obtained. Extensive numerical results are also provided.
\end{abstract}

Keywords: inhomogeneous birth and death processes, ergodicity bounds, perturbation bounds.

\section{Introduction}

This paper is devoted to time-dependent analysis of one subclass of continuous-time Markov chains, specifically, inhomogeneous birth and death processes with additional transitions from/to the origin. Suppose that the state space is $\mathcal{X}=\{0,1,2 \ldots\}$; then a transition, whenever it occurs

\footnotetext{
${ }^{*}$ Corresponding author
}

from state 0 , can be to any state $i>0$. Transition from state $i>0$ can be either to a neighbouring state $(i-1)$ or $(i+1)$, or to state 0 . All possible transition intensities are assumed to be non-random functions of time, and may depend (except for transition to state 0 ) on the state of the process. This subclass of processes is of particular interest in queueing theory for an analysis of queueing systems with catastrophes and bulk arrivals. It is well known that 
transient solutions, even for simple queueing models are more difficult to obtain than steady-state solutions and their importance is especially high in practical situations when the time horizon of a system's operation eventually terminates and/or remains finite.

A motivation for this research is due to the works of Parthasarathy and Kumar (1991) as well as Chen and Renshaw (1997). The former studied the transient behaviour of a time-homogeneous birth and death process with state space $\mathcal{X}$ and intensities dependent on the current process state. Being in state $i>0, i \in \mathcal{X}$, the process can move only either to neighbouring states or back to state $i$. But whenever the process arrives to state $i=0$, immigration can occur, i.e., the process can jump to any state $j>0, j \in \mathcal{X}$. With respect to queueing theory, this immigration is transition which occurs according to a homogeneous Poisson process with the size of arriving groups being independent and identically distributed random variables. For this birth and death process, the authors obtain an explicit expression for its state distribution at arbitrary time, asymptotic behaviour of extinction probability (eventually reaching state $i=0$ ) and its mean value.

Inspired by Parthasarathy and Kumar (1991), Chen and Renshaw (1997) introduced an $M / M / 1$ queueing model with the same type of immigration and possible catastrophes, which the authors call "mass arrivals when empty" and "mass exodus", respectively. Expressions were obtained for the number of customers in the system at arbitrary time and its moments. For Chen and Renshaw (1997) the term "mass exodus" means that, whenever a system is not empty, transition to an empty state occurs with intensity $\beta>0$. Having at hand the notion of negative customers (also known as signals or triggers), introduced by E. Gelenbe, one can say that there is an additional flow (independent of others) of customers that empty the system.

Since the publication of Chen and Renshaw (1997), some papers have appeared devoted to the study of birth and death processes with "mass exodus" and "mass arrivals when empty" (see, e.g., Chen et al., 2010; Chen and Renshaw, 2004; Li and Chen, 2013; Gaidamaka et al., 2014), and what they all have in common is that transition intensities are assumed to be constants independent of time. We tried to look further and see if the time-dependent analysis of such birth and death processes can be extended to the case when all possible transition intensities are non-random functions of time. Exact computation of a transient state probability distribution is not a very appealing way to treat these processes because it leads to a solution of an infinite system of differential equations and has well-known drawbacks. An alternative way is the construction of bounds for performance characteristics of interest which can be computed fast and are tight enough to make the results meaningful. In this paper, we follow the latter way and obtain first ergodicity and perturbation bounds for this subclass of continuous-time Markov processes. In our analysis we heavily rely on the methodology developed by Granovsky and Zeifman (2004), Zeifman (1995) and Zeifman et al. (2008; 2006), which is based on the logarithmic norm of linear operators and special transformations of the intensity matrix of the Markov chain governing the behaviour of the process.

The article is organized as follows. In the next section, a description of the subclass of birth and death processes under consideration and preliminary remarks are given. In Sections 3 and 4, it is explained how one can obtain ergodicity and perturbation bounds. Section 5 is devoted to a method of truncation that allows calculation of the limiting characteristics, and Section 6 provides extensive numerical results, illustrating the efficiency of the bounds. The conclusion contains a summary of the obtained results and directions of further research.

\section{Birth and death process}

In order to keep a connection with the results previously obtained by other authors, in what follows we will use terms of mass exodus and mass arrivals when empty in order to describe transitions to and from the origin.

Let the process $X(t), t \geq 0$, be an inhomogeneous continuous-time Markov chain with state space $\mathcal{X}=$ $\{0,1,2 \ldots\}$. Transition, whenever it occurs from state 0 , can be to any state $i>0$. Transition from state $i>0$ can be either to neighbouring states $(i-1)$ or $(i+1)$, or to state 0 . All possible transition intensities are assumed to be non-random functions of time, and may depend (except for transition to 0 ) on the process state. Denote by $p_{i j}(s, t)=\operatorname{Pr}\{X(t)=j \mid X(s)=i\}, i, j \geq 0,0 \leq$ $s \leq t$ transition probabilities of $X(t)$ and by $p_{i}(t)=$ $\operatorname{Pr}\{X(t)=i\}$ the probability that the Markov chain $X(t)$ is in state $i$ at time $t$. Let $\mathbf{p}(t)=\left(p_{0}(t), p_{1}(t), \ldots\right)^{T}$ be the probability distribution vector at time $t$. Then, for the discussed process $X(t)$, we assume that, for $j \neq i$

$$
\begin{aligned}
\operatorname{Pr} & (X(t+h)=j \mid X(t)=i) \\
& = \begin{cases}\lambda_{i}(t) h+\alpha_{i j}(t, h), & \text { if } j=i+1, i>0, \\
\mu_{i}(t) h+\alpha_{i j}(t, h), & \text { if } j=i-1, i>1, \\
\beta(t) h+\alpha_{i j}(t, h), & \text { if } j=0, i>1, \\
r_{j}(t) h+\alpha_{i j}(t, h), & \text { if } j \geq 1, i=0, \\
\left(\mu_{1}(t)+\beta(t)\right) h & \\
+\alpha_{i j}(t, h), & \text { if } j=i-1, i=1, \\
\alpha_{i j}(t, h) & \text { otherwise, }\end{cases}
\end{aligned}
$$

where all $\alpha_{i j}(t, h)$ are $o(h)$ uniformly in $i$, i.e., $\sup _{i}\left|\alpha_{i j}(t, h)\right|=o(h)$. Intensity functions $\beta(t)$ and $r_{j}(t), j \geq 1$, are henceforth called mass exodus and mass arrivals intensities. 
In the inhomogeneous case, we assume that all intensity functions are linear combinations of a finite number of nonnegative functions locally integrable on $[0, \infty)$. Then the corresponding intensity matrix is

$Q(t)=\left(\begin{array}{ccccccc}a_{00}(t) & r_{1}(t) & r_{2}(t) & r_{3}(t) & r_{4}(t) & \ldots & \ldots \\ \beta(t)+\mu_{1}(t) & a_{11}(t) & \lambda_{1}(t) & 0 & 0 & \ldots & \ldots \\ \beta(t) & \mu_{2}(t) & a_{22}(t) & \lambda_{2}(t) & 0 & \ldots & \ldots \\ \ldots & \ldots & \ldots & \ldots & \ldots & \ldots & \ldots \\ \beta(t) & 0 & \ldots & \mu_{r}(t) & a_{r r}(t) & \lambda_{r}(t) & \ldots \\ \ldots & \ldots & \ldots & \ldots & \ldots & \ldots & \ldots\end{array}\right)$

Let $a_{i j}(t)=q_{j i}(t)$ for $j \neq i$ and

$$
a_{i i}(t)=-\sum_{j \neq i} a_{j i}(t)=-\sum_{j \neq i} q_{i j}(t)
$$

According to the standard approach, which was applied by Granovsky and Zeifman (2004), Zeifman (1995) and Zeifman et al. (2008; 2006), we assume that the intensity matrix is essentially bounded, i.e.,

$$
\left|a_{i i}(t)\right| \leq L<\infty
$$

for almost all $t \geq 0$.

Probabilistic dynamics of the analysed process $X(t)$ are given by the forward Kolmogorov system,

$$
\frac{\mathrm{d} \mathbf{p}(t)}{\mathrm{d} t}=A(t) \mathbf{p}(t),
$$

where $A(t)=Q^{T}(t)$ is the transposed intensity matrix of the process. Throughout the paper, by $\|\cdot\|$ we denote the $l_{1}$-norm, i.e., $\|\mathbf{x}\|=\sum_{i}\left|x_{i}\right|$, and $\|B\|=\sup _{j} \sum_{i}\left|b_{i j}\right|$ for $B=\left(b_{i j}\right)_{i, j=0}^{\infty}$. Let $\Omega$ be the set of all stochastic vectors, i.e., $l_{1}$ - vectors with nonnegative coordinates and unit norm. Then, we have $\|A(t)\|=2 \sup _{k}\left|a_{k k}(t)\right| \leq 2 L$ for almost all $t \geq 0$. Hence, the operator function $A(t)$ from $l_{1}$ into itself is bounded for almost all $t \geq 0$ and locally integrable on $[0 ; \infty)$. Therefore, we can consider (3) as a differential equation in the space $l_{1}$ with a bounded operator.

It is well known (see Daleckij and Krein, 1975) that the Cauchy problem for the differential equation (3) has a unique solution for an arbitrary initial condition, and $\mathbf{p}(s) \in \Omega$ implies $\mathbf{p}(t) \in \Omega$ for $t \geq s \geq 0$.

Denote by $E(t, k)=E\{X(t) \mid X(0)=k\}$ the expected value (mean) of the process $X(t)$ at moment $t$ under the initial condition $X(0)=k$.

Recall that the process $X(t)$ is called weakly ergodic if $\left\|\mathbf{p}^{*}(t)-\mathbf{p}^{* *}(t)\right\| \rightarrow 0$ as $t \rightarrow \infty$ for any initial conditions $\mathbf{p}^{*}(0), \mathbf{p}^{* *}(0)$, where $\mathbf{p}^{*}(t)$ and $\mathbf{p}^{* *}(t)$ are the corresponding solutions of (3). The process $X(t)$ has the limiting mean $\varphi(t)$ if $\lim _{t \rightarrow \infty}(\varphi(t)-E(t, k))=0$ for any $k$.

\section{Ergodicity bounds}

Theorem 1. Let the mass exodus intensity be essential, i.e.,

$$
\int_{0}^{\infty} \beta(t) \mathrm{d} t=\infty
$$

Then, for any arrival and service intensities and any mass arrivals, the process $X(t)$ is weakly ergodic (in the uniform operator topology) and the following bound for the rate of convergence holds:

$$
\left\|\mathbf{p}^{*}(t)-\mathbf{p}^{* *}(t)\right\| \leq 2 \mathrm{e}^{-\int_{0}^{t} \beta(\tau) \mathrm{d} \tau}
$$

for any initial conditions $\mathbf{p}^{*}(0), \mathbf{p}^{* *}(0)$ and any $t \geq 0$.

Proof. We can apply the approach of Zeifman and Korotysheva (2012). Namely, rewrite the forward Kolmogorov equation (3) as

$$
\frac{\mathrm{d} \mathbf{p}(t)}{\mathrm{d} t}=A^{*}(t) \mathbf{p}(t)+\mathbf{g}(t), \quad t \geq 0,
$$

where $\mathbf{g}(t)=(\beta(t), 0,0, \ldots)^{T}, A^{*}(t)=\left(a_{i j}^{*}(t)\right)_{i, j=0}^{\infty}$ and

$$
a_{i j}^{*}(t)= \begin{cases}a_{00}(t)-\beta(t) & \text { if } j=i=0, \\ \mu_{1}(t) & \text { if } j=1, i=0, \\ 0 & \text { if } j>1, i=0, \\ a_{i j}(t) & \text { otherwise. }\end{cases}
$$

Recall the definition of the logarithmic norm of an operator function (see Van Doorn et al., 2010; Granovsky and Zeifman, 2004; Zeifman et al., 2006).

Let $B(t), t \geq 0$, be a one-parameter family of bounded linear operators on a Banach space $\mathcal{B}$, and let $I$ denote the identity operator. For a given $t \geq 0$, the number

$$
\gamma(B(t))_{\mathcal{B}}=\lim _{h \rightarrow+0} \frac{\|I+h B(t)\|-1}{h}
$$

is called the logarithmic norm of the operator $B(t)$.

If $\mathcal{B}$ is an $(N+1)$-dimensional vector space with $l_{1}$ norm such that the operator $B(t)$ is given by the matrix $B(t)=\left(b_{i j}(t)\right)_{i, j=0}^{N}, t \geq 0$, then the logarithmic norm of $B(t)$ can be found explicitly:

$$
\gamma(B(t))=\sup _{j}\left(b_{j j}(t)+\sum_{i \neq j}\left|b_{i j}(t)\right|\right), \quad t \geq 0 .
$$

On the other hand, the logarithmic norm of the operator $B(t)$ is related to the Cauchy operator $V(t, s)$ of the system

$$
\frac{\mathrm{d} \mathbf{x}(t)}{\mathrm{d} t}=B(t) \mathbf{x}(t), \quad t \geq 0
$$


in the following way:

$$
\gamma(B(t))_{\mathcal{B}}=\lim _{h \rightarrow+0} \frac{\|V(t+h, t)\|-1}{h}, \quad t \geq 0 .
$$

From the latter, one can deduce the following bounds of the Cauchy operator $V(t, s)$ :

$$
\|V(t, s)\|_{\mathcal{B}} \leq \mathrm{e}^{\int^{t} \gamma(B(\tau)) \mathrm{d} \tau}, \quad 0 \leq s \leq t
$$

Here we can find the exact value of the logarithmic norm of operator function $A^{*}(t)$, namely,

$$
\gamma\left(A^{*}(t)\right)=\sup _{i}\left(a_{i i}^{*}(t)+\sum_{j \neq i}\left|a_{j i}^{*}(t)\right|\right)=-\beta(t) .
$$

Let $U^{*}(t, s)$ be the Cauchy operator for Eqn. (6). Then we obtain

$$
\left\|U^{*}(t, s)\right\| \leq \mathrm{e}^{-\int_{s}^{t} \beta(\tau) \mathrm{d} \tau},
$$

and hence we have

$$
\left\|\mathbf{p}^{*}(t)-\mathbf{p}^{* *}(t)\right\| \leq \mathrm{e}^{-\int_{0}^{t} \beta(\tau) \mathrm{d} \tau}\left\|\mathbf{p}^{*}(0)-\mathbf{p}^{* *}(0)\right\|
$$

for any initial conditions $\mathbf{p}^{*}(0), \mathbf{p}^{* *}(0)$ and any $t \geq 0$, which implies our claim.

Now we study the existence of the limiting mean. To this end, we apply the approach of Zeifman et al. (2010) with modifications that concern only the way the estimates are made. Let $l_{1 E}=\left\{\mathbf{z}=\left(p_{0}, p_{1}, p_{2}, \ldots\right)\right\}$ be the space of sequences such that $\|\mathbf{z}\|_{1 E}=\sum_{k \geq 0} k\left|p_{k}\right|<\infty$. Consider the diagonal matrix $D_{k}$,

$$
D_{k}=\operatorname{diag}(\underbrace{k, k, k, \ldots, k}_{k+1}, k+1, k+2, \ldots) \text {, }
$$

and the corresponding space of sequences $l_{1 k}=$ $\left\{\mathbf{z}=\left(p_{0}, p_{1}, p_{2}, \ldots\right)\right\}$ such that $\|\mathbf{z}\|_{1 k}=\left\|D_{k} \mathbf{z}\right\|_{1}<\infty$. Then $\|\mathbf{z}\|_{1 E} \leq\|\mathbf{z}\|_{1 k}$ for any $k \in \mathbb{N}$.

Assume now that

$$
\sum_{j \geq 1} \frac{j r_{j+k}(t)}{k} \leq \rho \beta(t), \quad \frac{\lambda_{i+k}(t)}{k} \leq \rho \beta(t),
$$

for some $k \in \mathbb{N}$, almost all $t \geq 0$, any $i \geq 0$, and some $\rho \in(0,1)$. Then we can obtain the following estimate for the logarithmic norm of the operator function $A^{*}(t)$ in the

$$
\begin{aligned}
& l_{1 k} \text {-norm: } \\
& \gamma\left(A^{*}(t)\right)_{1 k} \\
& =\gamma\left(D_{k} A^{*}(t) D_{k}^{-1}\right)=\sup _{i}\left(a_{i i}^{*}(t)+\sum_{j \neq i}\left|\frac{d_{j}}{d_{i}} a_{j i}^{*}(t)\right|\right) \\
& \leq-\min \left(\beta(t)-\sum_{i \geq 1} \frac{i r_{k+i}(t)}{k}, \beta(t)-\frac{\lambda_{k}(t)}{k},\right. \\
& \left.\quad \beta(t)+\inf _{i \geq 1} \frac{\mu_{i+k}(t)-\lambda_{i+k}(t)}{i+k}\right) \\
& \leq-(1-\rho) \beta(t) .
\end{aligned}
$$

On the other hand, we have $\left\|\mathbf{e}_{j}-\mathbf{e}_{0}\right\|_{1 k}=k(1+j)$ for any $j$ and $k$ such that $k \geq j$. Hence, the following estimate holds:

$$
\begin{aligned}
|E(t, j)-E(t, 0)| & \leq \mathrm{e}^{-\int_{0}^{t}(1-\rho) \beta(\tau) \mathrm{d} \tau}\left\|\mathbf{e}_{j}-\mathbf{e}_{0}\right\|_{1 k} \\
& \leq k(1+j) \mathrm{e}^{-\int_{0}^{t}(1-\rho) \beta(\tau) \mathrm{d} \tau}
\end{aligned}
$$

for any initial condition $j$ and any $t \geq 0$.

Theorem 2. Under the assumptions of Theorem 11 let (12) hold for some $k \in \mathbb{N}$ and some $\rho \in(0,1)$. Then the process $X(t)$ has the limiting mean, say $\phi(t)=E(t, 0)$, and the following bound for the rate of convergence holds:

$$
|E(t, j)-E(t, 0)| \leq k(1+j) \mathrm{e}^{-\int_{0}^{t}(1-\rho) \beta(\tau) \mathrm{d} \tau}
$$

for any initial condition $X(0)=j$ and any $t \geq 0$.

\section{Perturbation bounds}

Let $\bar{X}(t), t \geq 0$, be a "perturbed" process with an intensity matrix $\bar{Q}(t)$ and a transposed intensity matrix $\bar{A}(t)$, where the perturbation matrix $\hat{A}(t)=A(t)-\bar{A}(t)$ is small in some sense. We will consider some bounds of stability of the main characteristics for the process $X(t)$ under such perturbations. Here we study the simplest situation of the exponential ergodicity of $X(t)$. Namely, we suppose that

$$
\mathrm{e}^{-\int_{s}^{t} \beta(u) \mathrm{d} u} \leq M e^{-a(t-s)},
$$

for any $s$ and $t$ such that $0 \leq s \leq t$. The next statement follows immediately from Theorem 1 of Zeifman and Korotysheva (2012), see also the general approach of Zeifman and Korolev (2014).

Theorem 3. Let the mass exodus intensity satisfy (16), and let perturbations be uniformly small, i.e.

$$
\|\hat{A}(t)\| \leq \varepsilon
$$


for almost all $t \geq 0$. Then

$$
\limsup _{t \rightarrow \infty}\|\mathbf{p}(t)-\overline{\mathbf{p}}(t)\| \leq \frac{\varepsilon(1+\log M)}{a}
$$

for any initial conditions $\mathbf{p}(0)$ and $\overline{\mathbf{p}}(0)$.

Corollary 1. Let all intensities be 1-periodic functions. Let $\int_{0}^{1} \beta(t) \mathrm{d} t \geq \theta^{*}>0$ instead of (16). Then the bound (18) holds for

$$
M=\mathrm{e}^{K}, \quad a=\theta^{*},
$$

where $K=\sup _{|t-s| \leq 1} \int_{s}^{t} \beta(\tau) \mathrm{d} \tau<\infty$.

Remark 1. Note that in Theorem 3 and Corollary 1 the structure of the perturbation matrix $\hat{A}(t)$ may be arbitrary.

The investigation of perturbation bounds for the mean is much more complicated. We assume now that the perturbed process has the same structure, i.e., $\bar{X}(t)$ is also an inhomogeneous birth and death process with the same mass exodus and mass arrivals intensities and, in addition to (12) and (17), we assume that

$$
\sum_{j \geq 1} \frac{j \hat{r}_{j+k}(t)}{k} \leq \varepsilon, \quad \frac{\hat{\lambda}_{i+k}(t)}{k} \leq \varepsilon,
$$

for almost all $t \geq 0$ and any $i$.

Let

$$
\frac{\mathrm{d} \overline{\mathbf{p}}(t)}{\mathrm{d} t}=\bar{A}^{*}(t) \overline{\mathbf{p}}(t)+\overline{\mathbf{g}}(t)
$$

be the respective system for the perturbed process, instead of (6). If we rewrite (6) in the form

$$
\frac{\mathrm{d} \mathbf{p}(t)}{\mathrm{d} t}=\bar{A}^{*}(t) \mathbf{p}(t)+\overline{\mathbf{g}}(t)+\hat{A}^{*}(t) \mathbf{p}(t)+\hat{\mathbf{g}}(t),
$$

then

$$
\begin{aligned}
\mathbf{p}(t)= & \bar{U}^{*}(t) \mathbf{p}(0)+\int_{0}^{t} \bar{U}^{*}(t, \tau) \overline{\mathbf{g}}(\tau) \mathrm{d} \tau \\
& +\int_{0}^{t} \bar{U}^{*}(t, \tau)\left(\hat{A}^{*}(\tau) \mathbf{p}(\tau)+\hat{\mathbf{g}}(\tau)\right) \mathrm{d} \tau
\end{aligned}
$$

and

$$
\overline{\mathbf{p}}(t)=\bar{U}^{*}(t) \overline{\mathbf{p}}(0)+\int_{0}^{t} \bar{U}^{*}(t, \tau) \overline{\mathbf{g}}(\tau) \mathrm{d} \tau .
$$

Hence, in any norm,

$$
\begin{aligned}
\|\mathbf{p}(t)-\overline{\mathbf{p}}(t)\| & \leq \int_{0}^{t}\left\|\bar{U}^{*}(t, \tau)\right\| \\
& \times\left(\left\|\hat{A}^{*}(\tau)\right\|\|\mathbf{p}(\tau)\|+\|\hat{\mathbf{g}}(\tau)\|\right) \mathrm{d} \tau
\end{aligned}
$$

if the initial conditions for the perturbed and original processes are the same.
Now, notice that

$$
\left\|\hat{A}^{*}(t)\right\|_{1 k} \leq 3 \varepsilon
$$

for almost any $t \geq 0$ in accordance with (17) and (20). Then we can write

$$
\begin{aligned}
\gamma\left(\bar{A}^{*}(t)\right)_{1 k} & \leq \gamma\left(D_{k} A^{*}(t) D_{k}^{-1}\right)_{1}+\left\|\hat{A}^{*}(t)\right\|_{1 k} \\
& \leq-(1-\rho) \beta(t)+3 \varepsilon .
\end{aligned}
$$

On the other hand, we have

$$
\begin{aligned}
& \|\mathbf{p}(t)\|_{1 k} \leq\left\|U^{*}(t) \mathbf{p}(0)\right\|_{1 k} \\
& +\int_{0}^{t}\left\|U^{*}(t, \tau) \mathbf{g}(\tau) \mathrm{d} \tau\right\|_{1 k} \\
& \leq \mathrm{e}^{\int^{t}(-(1-\rho) \beta(u)) \mathrm{d} u}\|\mathbf{p}(0)\|_{1 k}
\end{aligned}
$$

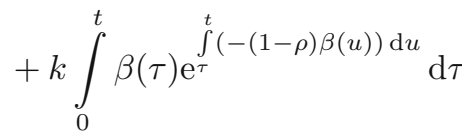

$$
\begin{aligned}
& \leq M^{1-\rho} \mathrm{e}^{-a(1-\rho) t}\|\mathbf{p}(0)\|_{1 k}+\frac{k}{1-\rho} \\
& \leq M^{1-\rho}\|\mathbf{p}(0)\|_{1 k}+\frac{k}{1-\rho}
\end{aligned}
$$

for any $\mathbf{p}(0)$, because $\|\mathbf{g}(\tau)\|_{1 k}=k \beta(\tau)$.

Finally, we obtain

$$
\begin{aligned}
& |E(t, j)-\bar{E}(t, j)| \\
& \leq\|\mathbf{p}(t)-\overline{\mathbf{p}}(t)\|_{1 k} \\
& \leq\left(3 \varepsilon\left(M^{1-\rho}\|\mathbf{p}(0)\|_{1 k}+\frac{k}{1-\rho}\right)+k \varepsilon\right) \\
& \quad \times \int_{0}^{t} \mathrm{e}^{-\int_{\tau}^{t}((1-\rho) \beta(u)-3 \varepsilon) \mathrm{d} u} \mathrm{~d} \tau \\
& \leq \frac{\varepsilon M^{1-\rho}}{a(1-\rho)-3 \varepsilon}\left(3\left(j k M^{1-\rho}+\frac{k}{1-\rho}\right)+k\right),
\end{aligned}
$$

where $\mathbf{p}(0)=\overline{\mathbf{p}}(0)=\mathbf{e}_{j}$, and the following theorem holds.

Theorem 4. Under the assumptions of Theorem 2 let the perturbations be such that (17) and (20) hold. Then

$$
\begin{aligned}
& \limsup _{t \rightarrow \infty}|E(t, j)-\bar{E}(t, j)| \\
& \quad \leq \frac{\varepsilon k M^{1-\rho}}{a(1-\rho)-3 \varepsilon}\left(3 j M^{1-\rho}+\frac{3}{1-\rho}+1\right)
\end{aligned}
$$

for any initial condition $X(0)=j$ and any $t \geq 0$.

\section{Truncations}

For the calculation of the limiting characteristics of processes $X(t)$ and $\bar{X}(t)$, we apply the approach of 
Zeifman et al. (2006), because the structure of the infinitesimal matrix does not allow us to use the uniform bounds and another approach of Zeifman et al. (2014). In this section, we additionally assume that there exists $q>1$ such that $r_{k}(t) \leq q^{-k}$ for any $k$.

Consider a "truncated" process $X_{n}(t)$ on the state space $E_{n}=\{0,1, \ldots, n\}$ with the corresponding reduced intensity matrix $A_{n}^{*}(t)$. Below we will identify the finite vector with entries, say, $\left(a_{1}, \ldots, a_{n}\right)^{T}$, and the infinite vector with the same first $n$ coordinates and the others equal to zero. Let us rewrite the system (6) as

$$
\frac{\mathrm{d} \mathbf{p}(t)}{\mathrm{d} t}=A_{n}^{*}(t) \mathbf{p}(t)+\mathbf{g}(t)+\left(A^{*}(t)-A_{n}^{*}(t)\right) \mathbf{p}(t),
$$

and consider the corresponding "truncated" system

$$
\frac{\mathrm{d} \mathbf{p}_{n}(t)}{\mathrm{d} t}=A_{n}^{*}(t) \mathbf{p}_{n}(t)+\mathbf{g}(t)
$$

In any norm, we can write

$$
\begin{aligned}
& \left\|\mathbf{p}(t)-\mathbf{p}_{n}(t)\right\| \\
& =\left\|\int_{0}^{t} U_{n}^{*}(t, \tau)\left(A^{*}(\tau)-A_{n}^{*}(\tau)\right) \mathbf{p}(\tau) \mathrm{d} \tau\right\| .
\end{aligned}
$$

Consider the corresponding Cauchy matrix

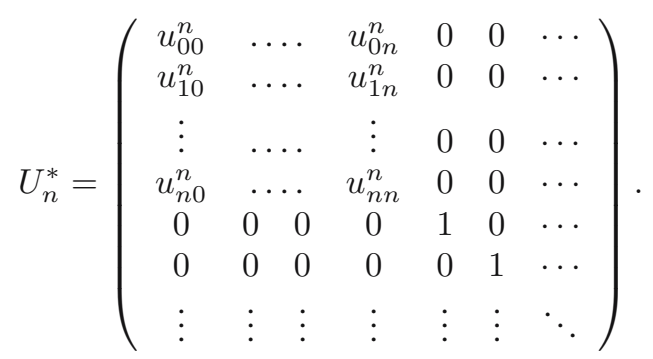

For the sake of brevity, we will omit the argument $t$ till the end of this section. Having noticed that

$$
\begin{aligned}
& \left(A^{*}-A_{n}^{*}\right) \mathbf{p} \\
& =\left(0, \ldots, \mu_{n+1} p_{n+1}, r_{n+1} p_{0}\right. \\
& \left.\quad+\lambda_{n} p_{n}-\left(\lambda_{n+1}+\mu_{n+1}+\beta\right) p_{n+1}+\mu_{n+2} p_{n+2}, \ldots\right)^{T},
\end{aligned}
$$

we obtain

$$
U_{n}^{*}\left(A^{*}-A_{n}^{*}\right) \mathbf{p}=\left(\begin{array}{c}
u_{0 n}^{n} \mu_{n+1} p_{n+1} \\
u_{1 n}^{n} \mu_{n+1} p_{n+1} \\
\vdots \\
u_{n n}^{n} \mu_{n+1} p_{n+1} \\
{\left[r_{n+1} p_{0}+\lambda_{n} p_{n}\right.} \\
-\left(\lambda_{n+1}+\mu_{n+1}+\beta\right) p_{n+1} \\
\left.+\mu_{n+2} p_{n+2}\right] \\
\vdots
\end{array}\right) .
$$

The inequalities $u_{i j}^{n}(t, \tau) \geq 0$ (for any $\left.i, j, t, \tau\right)$ and the inequalities $\sum_{i} u_{i j}^{n}(t, \tau) \leq 1$ (for any $j, t, \tau$ ) imply the bounds

$$
\begin{aligned}
& \left\|U_{n}^{*}\left(A^{*}-A_{n}^{*}\right) \mathbf{p}\right\| \\
& \leq\left|\mu_{n+1} p_{n+1}\right| \sum_{k \leq n} u_{k n}^{n}+\sum_{k \geq n} \mid r_{k+1} p_{0}+\lambda_{n} p_{k} \\
& \quad-\left(\lambda_{n+1}+\mu_{n+1}+\beta\right) p_{k+1}+\mu_{n+2} p_{k+2} \mid \\
& \leq \sum_{k \geq n}\left|r_{k}\right| p_{0}+2 L \sum_{k \geq n+1} p_{k}+L \sum_{k \geq n+1} p_{k}+2 L \sum_{k \geq n} p_{k} \\
& \leq \frac{1}{(q-1) q^{n-1}}+5 L \sum_{k \geq n} p_{k} \\
& \leq \frac{1}{(q-1) q^{n-1}}+\frac{5 L}{n} \sum_{k \geq n} k p_{k}
\end{aligned}
$$

and

$$
\begin{aligned}
\| & U_{n}^{*}\left(A^{*}-A_{n}^{*}\right) \mathbf{p} \|_{1 E} \\
= & \left|\mu_{n+1} p_{n+1}\right| \sum_{k \leq n} k u_{k n}^{n}+\sum_{k \geq n}(k+1) \mid r_{k+1} p_{0}+\lambda_{n} p_{k} \\
& -\left(\lambda_{n+1}+\mu_{n+1}+\beta\right) p_{k+1}++\mu_{n+2} p_{k+2} \mid \\
\leq & \frac{n}{(q-1) q^{n}}+\frac{1}{(q-1)^{2} q^{n-1}}+L \sum_{k \geq n}(2 k+1) p_{k} \\
\leq & \frac{n+1}{(q-1)^{2} q^{n-1}}+\frac{3 L(n+1)}{n} \sum_{k \geq n} k p_{k} .
\end{aligned}
$$

On the other hand, we have

$$
\begin{aligned}
& d_{n} p_{n}+d_{n+1} p_{n+1}+\cdots \\
& =\frac{d_{n}}{n} n p_{n}+\frac{d_{n+1}}{n+1} p_{n+1}+\cdots \geq \inf _{i \geq n} \frac{d_{i}}{i} \sum_{k \geq n} k p_{k}
\end{aligned}
$$

and

$$
\begin{aligned}
\sum_{k \geq n} k p_{k} & \leq \frac{1}{\inf _{i \geq n} \frac{d_{i}}{i}} \sum_{k \geq n} d_{n} p_{n} \\
& \leq \frac{1}{\inf _{i \geq n} \frac{d_{i}}{i}}\|\mathbf{p}\|_{1 D} \leq \frac{d_{0}}{\inf _{i \geq n} \frac{d_{i}}{i}} .
\end{aligned}
$$

Therefore, we get the following bounds of truncation:

$$
\begin{gathered}
\left\|\mathbf{p}(t)-\mathbf{p}_{n}(t)\right\| \leq \frac{1}{(q-1) q^{n-1}}+\frac{5 L d_{0} t}{n \inf _{i \geq n} \frac{d_{i}}{i}}, \\
\left|E_{\mathbf{p}}(t)-E_{\mathbf{p}_{\mathbf{n}}}(t)\right| \leq \frac{n+1}{(q-1)^{2} q^{n-1}}+\frac{3 L d_{0}(n+1) t}{n \inf _{i \geq n} \frac{d_{i}}{i}} .
\end{gathered}
$$

In the next section, we proceed to numerical examples, illustrating the efficiency of the obtained bounds. 


\section{Examples}

The efficiency of the bounds obtained for the process $X(t)$ in the previous sections will be illustrated in the queueing theory context. Specifically, we consider a $M_{t} / M_{t} / S$ queue with catastrophes and bulk arrivals when empty when intensities are periodic functions of time which can be described by the process $X(t)$. In each example it is shown how to find approximations for the limiting value of the mean number of customers in the system and the limiting value of an empty system with a given error. For convenience, we first give a detailed description of the system and then proceed to examples.

The queueing system consists of a single infinite capacity queue and $S$ servers. Two flows of customers arrive at the system: a flow of ordinary customers and a flow of catastrophes. If at time $t$ there is at least one customer in the system, then new arrivals of ordinary customers happen according to an inhomogeneous Poisson process with intensity $\lambda(t)$. But if at time $t$ the system is empty, ordinary customers arrive in bulk (or groups) in accordance with an inhomogeneous Poisson process of intensity $r(t)$. The size of an arriving group is a random variable with a probability distribution $g_{n}, n=1,2, \ldots$, having a finite mean. The sizes and interarrival times of successive arriving groups are stochastically independent.

Let $r_{n}(t)=g_{n} r(t)$. Each ordinary customer upon arrival occupies one place in the queue and waits for service. Whenever the server becomes free, a customer from the queue (if there is any) enters the server and gets served according to the exponential distribution with intensity $\mu(t)$ (service discipline is unimportant, and for certainty one can assume that the customers are served in a FIFO manner). An additional inhomogeneous Poisson flow of catastrophes of intensity $\beta(t)$ arrives at the system. If an arriving customer of this flow finds the system busy, it removes all customers from the system and leaves it. Otherwise, it has no effect on it.

The only non-trivial cases are those when there are few servers in the system and when there are many servers in the system. They are considered in what follows.

6.1. Case when $S=3$. Let the number of servers in the system be equal to $S=3$. We will consider four examples, which differ in periods of transition intensities.

Example 1. Let the intensities have the form

$$
\begin{aligned}
\lambda_{n}(t) & =\lambda(t)=1+\sin 2 \pi t, \\
\mu_{n}(t) & =\min (n, S) \mu(t)=\min (n, 3)(1-\sin 2 \pi t), \\
\beta(t) & =2+\cos 2 \pi t, \\
r_{n}(t) & =\frac{1+\sin 2 \pi t}{4^{n}} .
\end{aligned}
$$

Then the process $X(t)$ is weakly ergodic, has the limiting 1-periodic regime, and the bound

$$
\left\|\mathbf{p}^{*}(t)-\mathbf{p}^{* *}(t)\right\| \leq 2 \mathrm{e}^{-\int_{0}^{t} \beta(\tau) \mathrm{d} \tau} \leq 4 e^{-2 t}
$$

holds for any initial conditions $\mathbf{p}^{*}(0), \mathbf{p}^{* *}(0)$ and any $t \geq$ 0 . Moreover, the inequality (12) holds for $k=2, \rho=$ 0.5 . Hence, $X(t)$ has a limiting (1-periodic) mean with the bound

$$
|E(t, j)-E(t, 0)| \leq 3(j+1) e^{-t}
$$

for any initial condition $j$ and any $t \geq 0$. We now have $M=e^{1 / \pi}$ and $a=2$, and thus the perturbation bound

$$
\limsup _{t \rightarrow \infty}\|\mathbf{p}(t)-\overline{\mathbf{p}}(t)\| \leq \frac{\varepsilon(1+\log M)}{a}<\varepsilon
$$

holds for any initial conditions $\mathbf{p}(0)$ and $\overline{\mathbf{p}}(0)$, and for any structure of the perturbed intensity matrix.

If the perturbed intensity matrix has a certain structure (e.g., the relation (20) holds), then the perturbation bound can be made more precise:

$$
\limsup _{t \rightarrow \infty}|E(t, j)-\bar{E}(t, j)|<\frac{\varepsilon(9 j+17)}{1-3 \varepsilon}
$$

for any $j$

Setting $d_{n}=2^{n-1}$, applying this approach and using the obtained bounds, we can find the approximation to the limiting mean of the queue, and the limiting probability of the empty queue with a truncation error of $10^{-5}$ for $n=35$ and $t \in[15,16]$ (see Figs 144).

Example 2. Let the intensities have the form

$$
\begin{aligned}
\lambda_{n}(t) & =\lambda(t)=1+\sin 20 \pi t, \\
\mu_{n}(t) & =\min (n, S) \mu(t)=\min (n, 3)(1-\sin 20 \pi t), \\
\beta(t) & =2+\cos 20 \pi t, \\
r_{n}(t) & =\frac{1+\sin 20 \pi t}{4^{n}} .
\end{aligned}
$$

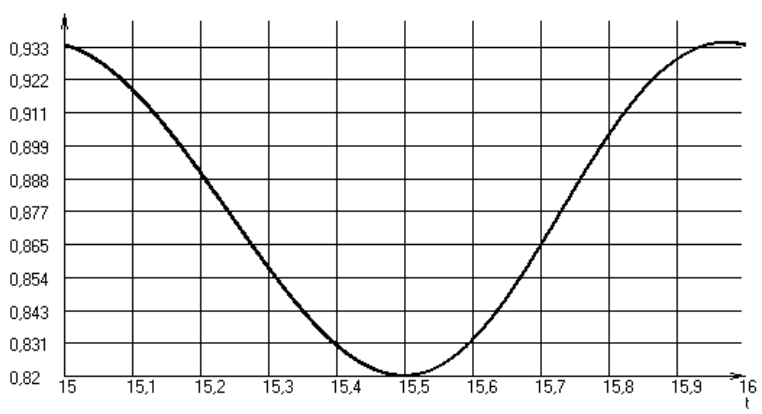

Fig. 1. Example 1: approximation of the limiting probability of empty queue $\operatorname{Pr}\{\bar{X}(t)=0 \mid \bar{X}(0)=0\}$ on $[15,16]$. 
Then the process $X(t)$ is weakly ergodic, has the limiting 0.1-periodic regime and the bound

$$
\left\|\mathbf{p}^{*}(t)-\mathbf{p}^{* *}(t)\right\| \leq 2 \mathrm{e}^{-\int_{0}^{t} \beta(\tau) \mathrm{d} \tau} \leq 4 \mathrm{e}^{-2 t}
$$

holds for any initial conditions $\mathbf{p}^{*}(0), \mathbf{p}^{* *}(0)$ and any $t \geq$ 0 . The inequality (12) holds for $k=2, \rho=0.5$, as well as in Example 1. Hence $X(t)$ has the limiting (0.1-periodic) mean with bound

$$
|E(t, j)-E(t, 0)| \leq 3(j+1) \mathrm{e}^{-t}
$$

for any initial condition $j$ and any $t \geq 0$. We have that

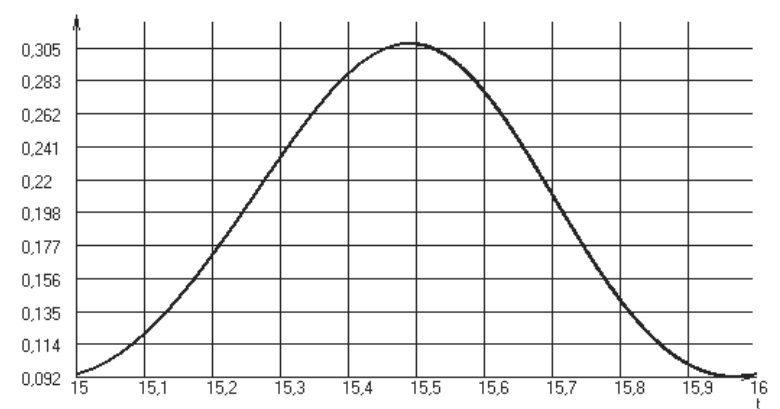

Fig. 2. Example 1: approximation of the limiting mean $\bar{E}(t, 0)$ on $[15,16]$.

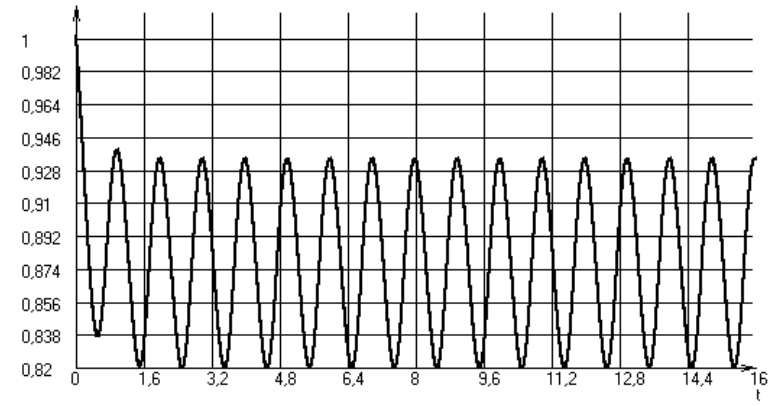

Fig. 3. Example 1: approximation of the probability of the empty queue $\operatorname{Pr}\{\bar{X}(t)=0 \mid \bar{X}(0)=0\}$ on $[0,16]$.

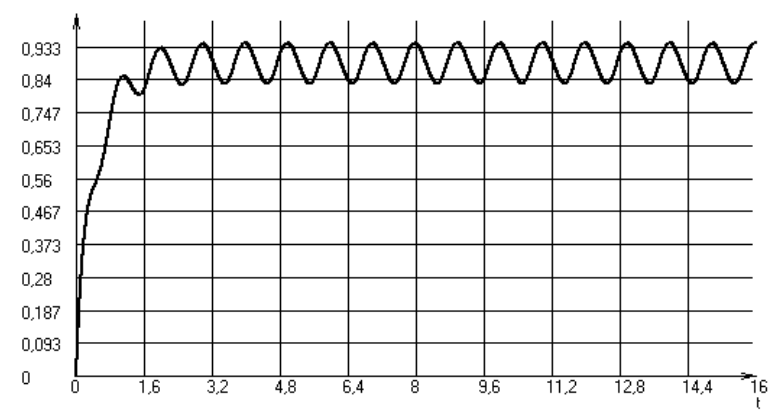

Fig. 4. Example 1: approximation of the probability of the empty queue $\operatorname{Pr}\{\bar{X}(t)=0 \mid \bar{X}(0)=0\}$ on $[0,16]$.
$M=e^{1 / 10 \pi}$ and $a=2$. Thus the perturbation bound

$$
\limsup _{t \rightarrow \infty}\|\mathbf{p}(t)-\overline{\mathbf{p}}(t)\| \leq \frac{\varepsilon(1+\log M)}{a}<\varepsilon
$$

is valid for any initial conditions $\mathbf{p}(0)$ and $\overline{\mathbf{p}}(0)$, and for any structure of the perturbed intensity matrix.

If the perturbed intensity matrix has a certain structure (e.g., the relation (20) holds), then the perturbation bound is

$$
\limsup _{t \rightarrow \infty}|E(t, j)-\bar{E}(t, j)|<\frac{\varepsilon(7 j+15)}{1-3 \varepsilon}
$$

for any $j$.

Setting $d_{n}=2^{n-1}$, applying this approach and using the obtained bounds, we can find the approximation of the limiting mean of the queue, and the limiting probability of the empty queue with a truncation error of $10^{-5}$ for $n=35$ and $t \in[15,15.1]$ (see Figs. 5, 8 ).

Example 3. Let the intensities have the form

$$
\begin{aligned}
\lambda_{n}(t) & =\lambda(t)=1+\sin 0.2 \pi t, \\
\mu_{n}(t) & =\min (n, S) \mu(t)=\min (n, 3)(1-\sin 0.2 \pi t), \\
\beta(t) & =2+\cos 0.2 \pi t, \\
r_{n}(t) & =\frac{1+\sin 0.2 \pi t}{4^{n}} .
\end{aligned}
$$

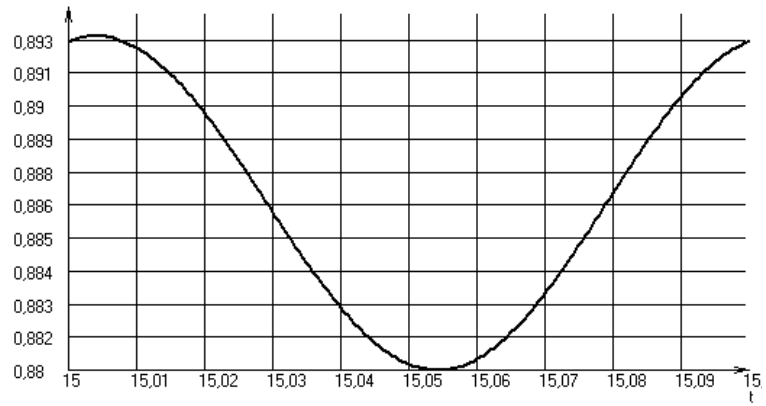

Fig. 5. Example 2: approximation of the limiting probability of empty queue $\operatorname{Pr}\{\bar{X}(t)=0 \mid \bar{X}(0)=0\}$ on $[15,15.1]$.

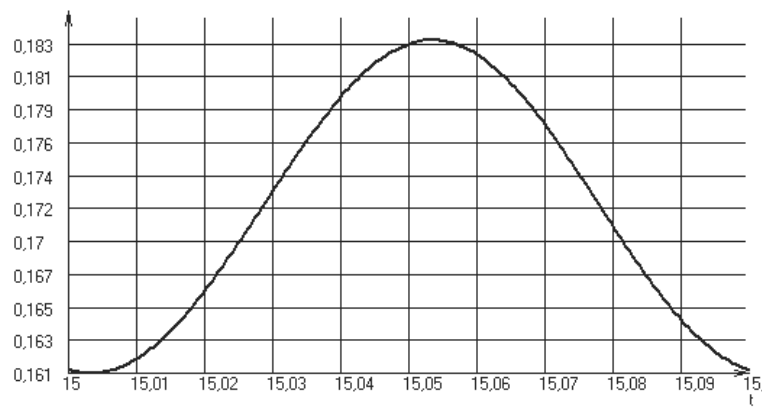

Fig. 6. Example 2: approximation of the limiting mean $\bar{E}(t, 0)$ on $[15,15.1]$. 
Then the process $X(t)$ is weakly ergodic, has the limiting 10-periodic regime, and the bound

$$
\left\|\mathbf{p}^{*}(t)-\mathbf{p}^{* *}(t)\right\| \leq 2 \mathrm{e}^{-\int_{0}^{t} \beta(\tau) \mathrm{d} \tau} \leq 10 \mathrm{e}^{-2 t}
$$

holds for any initial conditions $\mathbf{p}^{*}(0), \mathbf{p}^{* *}(0)$ and any $t \geq$ 0 . Moreover, the inequality (12) holds for $k=2, \rho=0.5$, and thus $X(t)$ has the limiting (10-periodic) mean with bound

$$
|E(t, j)-E(t, 0)| \leq 5(j+1) \mathrm{e}^{-t}
$$

for any initial condition $j$ and any $t \geq 0$.

We have $M=\mathrm{e}^{10 / \pi}$ and $a=2$, and the perturbation bound

$$
\limsup _{t \rightarrow \infty}\|\mathbf{p}(t)-\overline{\mathbf{p}}(t)\| \leq \frac{\varepsilon(1+\log M)}{a}<6 \varepsilon
$$

holds for any initial conditions $\mathbf{p}(0)$ and $\overline{\mathbf{p}}(0)$, and for any structure of the perturbed intensity matrix.

If the perturbed intensity matrix has a certain structure (e.g., 20 holds), then, in addition, the perturbation bound

$$
\limsup _{t \rightarrow \infty}|E(t, j)-\bar{E}(t, j)|<\frac{\varepsilon(145 j+69)}{1-3 \varepsilon}
$$

holds for any $j$.

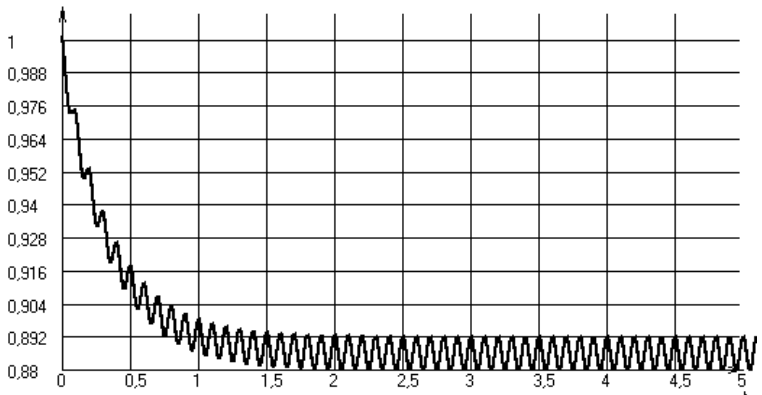

Fig. 7. Example 2: approximation of the probability of the empty queue $\operatorname{Pr}\{\bar{X}(t)=0 \mid \bar{X}(0)=0\}$ on $[0,5]$

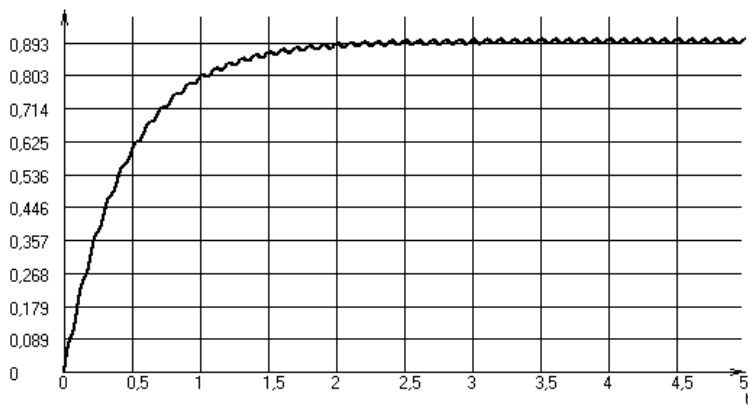

Fig. 8. Example 2: approximation of the probability of the empty queue $\operatorname{Pr}\{\bar{X}(t)=0 / \bar{X}(0)=35\}$ on $[0,5]$.
Setting $d_{n}=2^{n-1}$, applying this approach and using the obtained bounds, we can find an approximation to the limiting mean of the queue, and the limiting probability of the empty queue with a truncation error of $10^{-5}$ for $n=35$ and $t \in[15,25]$ (see Figs. 9] $[12$ ).

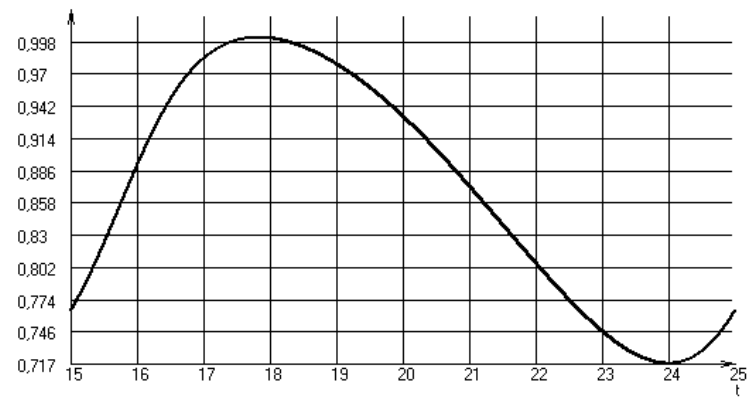

Fig. 9. Example 3: approximation of the limiting probability of the empty queue $\operatorname{Pr}\{\bar{X}(t)=0 \mid \bar{X}(0)=0\}$ on $[15,25]$.

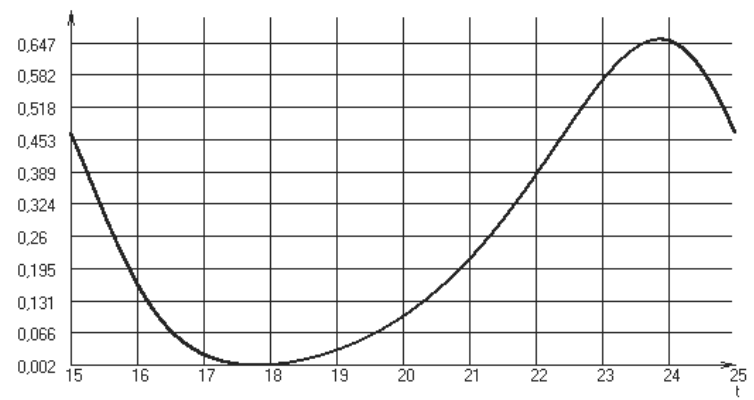

Fig. 10. Example 3: approximation of the limiting mean $\bar{E}(t, 0)$ on $[15,25]$.

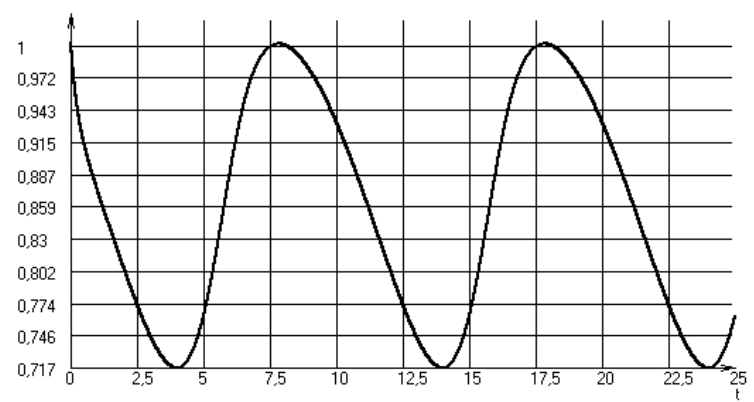

Fig. 11. Example 3: approximation of the probability of the empty queue $\operatorname{Pr}\{\bar{X}(t)=0 \mid \bar{X}(0)=0\}$ on $[0,25]$. 
Example 4. Let the intensities have the form

$$
\begin{aligned}
\lambda_{n}(t) & =\lambda(t)=1+0.5 \sin 2 \pi t+0.5 \sin 20 \pi, \\
\mu_{n}(t) & =\min (n, S) \mu(t) \\
& =\min (n, 3)(1-0.5 \sin 2 \pi t-0.5 \sin 20 \pi t), \\
\beta(t) & =2+0.5 \cos 2 \pi t+0.5 \cos 20 \pi, \\
r_{n}(t) & =\frac{1+0.5 \sin 2 \pi t+0.5 \sin 20 \pi t}{4^{n}} .
\end{aligned}
$$

Then the process $X(t)$ is weakly ergodic, has the limiting 1-periodic regime, and the bound

$$
\left\|\mathbf{p}^{*}(t)-\mathbf{p}^{* *}(t)\right\| \leq 2 \mathrm{e}^{-\int_{0}^{t} \beta(\tau) \mathrm{d} \tau} \leq 4 \mathrm{e}^{-2 t}
$$

holds for any initial conditions $\mathbf{p}^{*}(0), \mathbf{p}^{* *}(0)$ and any $t \geq$ 0 . Moreover, the inequality (12) is fulfilled for $k=2$, $\rho=0.5$. Hence $X(t)$ has the limiting (1-periodic) mean and the bound

$$
|E(t, j)-E(t, 0)| \leq 3(j+1) e^{-t}
$$

holds for any initial condition $j$ and any $t \geq 0$.

Now we have $M=\mathrm{e}^{1 / 2 \pi+1 / 20 \pi}$ and $a=2$; hence, the corresponding perturbation bound

$$
\limsup _{t \rightarrow \infty}\|\mathbf{p}(t)-\overline{\mathbf{p}}(t)\| \leq \frac{\varepsilon(1+\log M)}{a}<\varepsilon
$$

holds for any initial conditions $\mathbf{p}(0)$ and $\overline{\mathbf{p}}(0)$, and for any structure of the perturbed intensity matrix.

If the perturbed intensity matrix has a certain structure (e.g., 20 holds), then, in addition, the perturbation bound

$$
\limsup _{t \rightarrow \infty}|E(t, j)-\bar{E}(t, j)|<\frac{\varepsilon(8 j+16)}{1-3 \varepsilon}
$$

holds for any $j$.

Set $d_{n}=2^{n-1}$; then, applying this approach and using the obtained bounds, we can find the approximation of the limiting mean of the queue, and the limiting probability of the empty queue with a truncation error of $10^{-5}$ for $n=35$ and $t \in[15,16]$ (see Figs. 13 16).

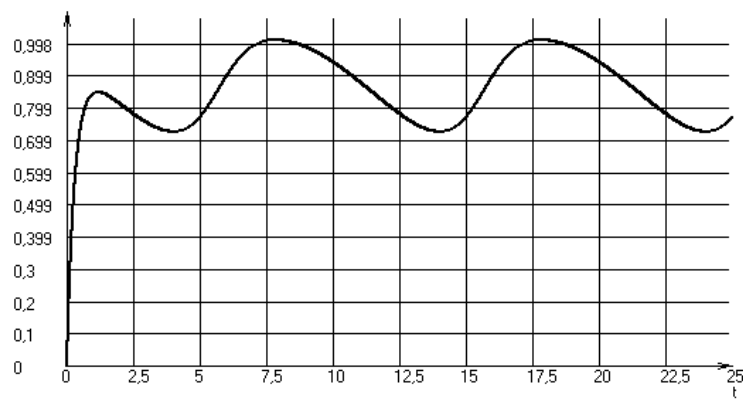

Fig. 12. Example 3: approximation of the probability of the empty queue $\operatorname{Pr}\{\bar{X}(t)=0 / \bar{X}(0)=35\}$ on $[0,25]$.
6.2. Case when $S=1000$. Let the number of servers in the system be equal to $S=1000$. Here we will consider again four examples as in the previous subsection, which differ in periods of transition intensities.

Example 5. Let the intensities have the form

$$
\begin{aligned}
\lambda_{n}(t) & =\lambda(t)=10^{3}(1+\sin 2 \pi t), \\
\mu_{n}(t) & =\min (n, S) \mu(t) \\
& =\min (n, 1000)(1-\sin 2 \pi t),
\end{aligned}
$$

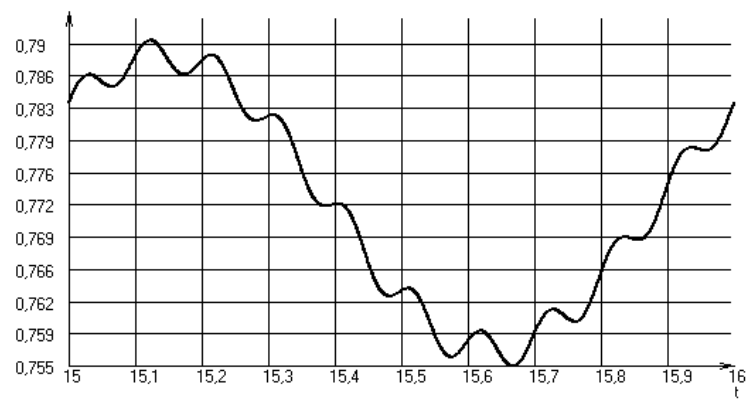

Fig. 13. Example 4: approximation of the limiting probability of the empty queue $\operatorname{Pr}\{\bar{X}(t)=0 \mid \bar{X}(0)=0\}$ on $[15,16]$.

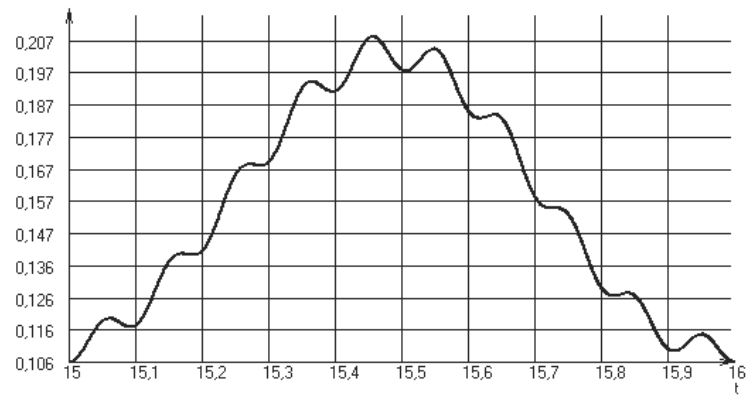

Fig. 14. Example 4: approximation of the limiting mean $\bar{E}(t, 0)$ on $[15,16]$.

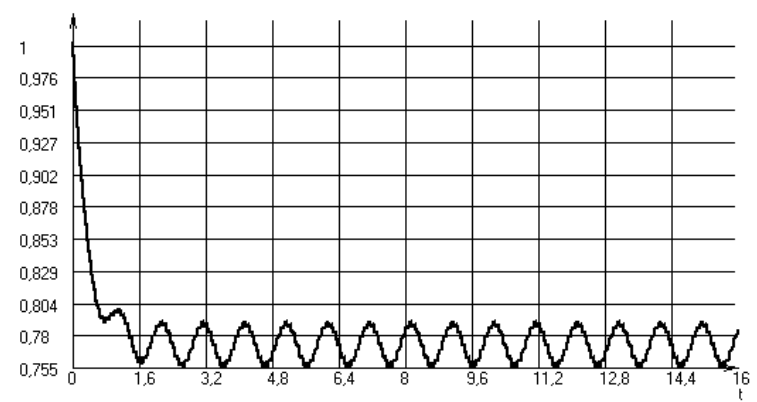

Fig. 15. Example 4: approximation of the probability of the empty queue $\operatorname{Pr}\{\bar{X}(t)=0 \mid \bar{X}(0)=0\}$ on $[0,16]$. 


$$
\begin{aligned}
\beta(t) & =2+\cos 2 \pi t \\
r_{n}(t) & =\frac{1+\sin 2 \pi t}{4^{n}}
\end{aligned}
$$

Then the process $X(t)$ is weakly ergodic, has the limiting 1-periodic regime, and the bound

$$
\left\|\mathbf{p}^{*}(t)-\mathbf{p}^{* *}(t)\right\| \leq 2 \mathrm{e}^{-\int_{0}^{t} \beta(\tau) \mathrm{d} \tau} \leq 4 \mathrm{e}^{-2 t}
$$

holds for any initial conditions $\mathbf{p}^{*}(0), \mathbf{p}^{* *}(0)$ and any $t \geq$ 0 . Moreover, the inequality (12) holds for $k=2 \cdot 10^{3}$, $\rho=0.5$, hence $X(t)$ has the limiting $(1-$ periodic $)$ mean, and the bound

$$
|E(t, j)-E(t, 0)| \leq 3 \cdot 10^{3}(j+1) \mathrm{e}^{-t}
$$

holds for any initial condition $j$ and any $t \geq 0$. Now we have $M=\mathrm{e}^{1 / \pi}$ and $a=2$; hence the corresponding perturbation bound

$$
\limsup _{t \rightarrow \infty}\|\mathbf{p}(t)-\overline{\mathbf{p}}(t)\| \leq \frac{\varepsilon(1+\log M)}{a}<\varepsilon,
$$

holds for any initial conditions $\mathbf{p}(0)$ and $\overline{\mathbf{p}}(0)$, and for any structure of perturbed intensity matrix.

If the perturbed intensity matrix has a certain structure (e.g., 20) holds), then, in addition, the perturbation bound

$$
\limsup _{t \rightarrow \infty}|E(t, j)-\bar{E}(t, j)|<\frac{10^{3} \varepsilon(9 j+17)}{1-3 \varepsilon}
$$

holds for any $j$.

Set $d_{n}=4^{n-1}$. Then applying this approach and using the obtained bounds, we can find an approximation to the limiting mean of the queue, and the limiting probability of the empty queue with a truncation error of $10^{-5}$ for $n=22$ and $t \in[23,24]$ (see Figs. 17 20).

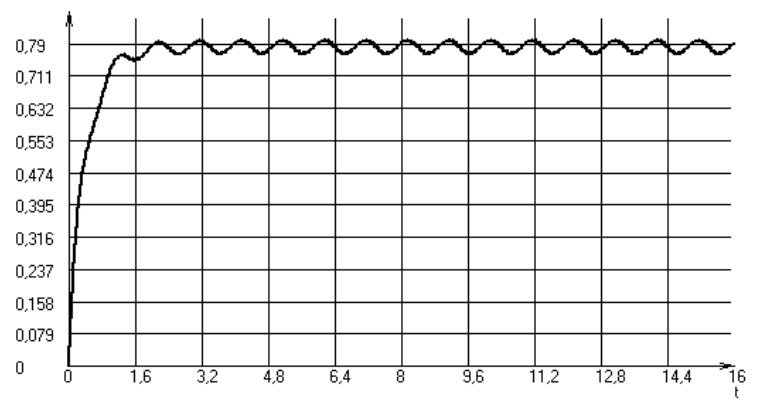

Fig. 16. Example 4: approximation of the probability of the empty queue $\operatorname{Pr}\{\bar{X}(t)=0 / \bar{X}(0)=35\}$ on $[0,16]$.
Example 6. Let the intensities have the form

$$
\begin{aligned}
\lambda_{n}(t) & =\lambda(t)=10^{3}(1+\sin 20 \pi t), \\
\mu_{n}(t) & =\min (n, S) \mu(t) \\
& =\min (n, 1000)(1-\sin 20 \pi t), \\
\beta(t) & =2+\cos 20 \pi t, \\
r_{n}(t) & =\frac{1+\sin 20 \pi t}{4^{n}} .
\end{aligned}
$$

Then the process $X(t)$ is weakly ergodic, has the

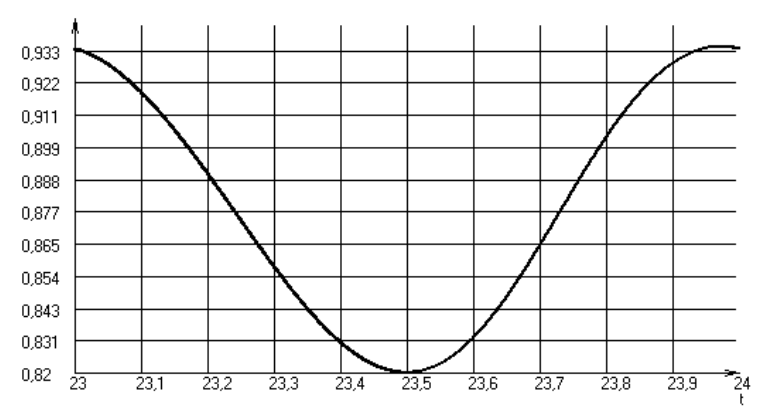

Fig. 17. Example 5: approximation of the limiting probability of the empty queue $\operatorname{Pr}\{\bar{X}(t)=0 \mid \bar{X}(0)=0\}$ on $[23,24]$.

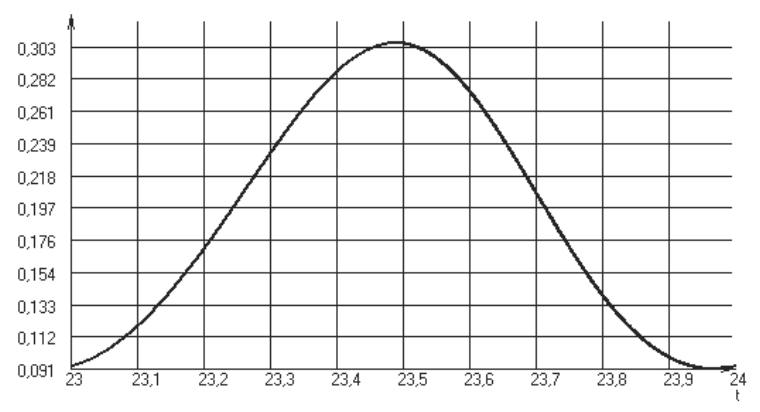

Fig. 18. Example 5: approximation of the limiting mean $E(t, 0)$ on $[23,24]$.

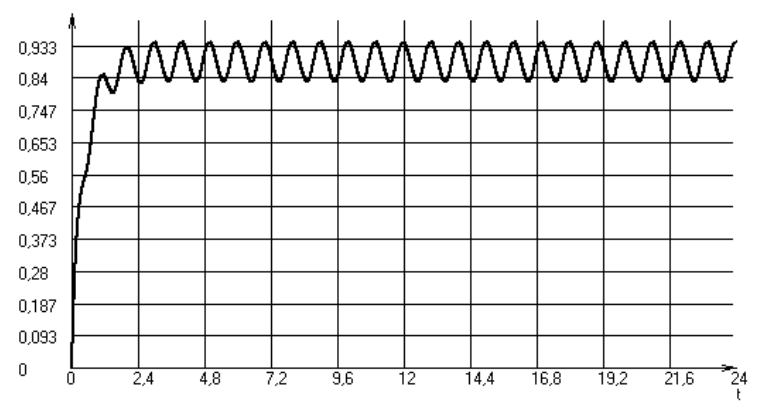

Fig. 19. Example 5: approximation of the probability of the empty queue $\operatorname{Pr}\{\bar{X}(t)=0 / \bar{X}(0)=22\}$ on $[0,24]$. 
limiting 0.1-periodic regime, and the bound

$$
\left\|\mathbf{p}^{*}(t)-\mathbf{p}^{* *}(t)\right\| \leq 2 \mathrm{e}^{-\int_{0}^{t} \beta(\tau) d \tau} \leq 4 \mathrm{e}^{-2 t}
$$

holds for any initial conditions $\mathbf{p}^{*}(0), \mathbf{p}^{* *}(0)$ and any $t \geq$ 0 . Moreover, the inequality (12) is fulfilled for $k=2 \cdot 10^{3}$, $\rho=0.5$ hence $X(t)$ has the limiting $(0.1$ - periodic $)$ mean, and the bound

$$
|E(t, j)-E(t, 0)| \leq 3 \cdot 10^{3}(j+1) \mathrm{e}^{-t}
$$

holds for any initial condition $j$ and any $t \geq 0$. Here we have $M=\mathrm{e}^{1 / 10 \pi}$ and $a=200$. Hence the corresponding perturbation bound

$$
\limsup _{t \rightarrow \infty}\|\mathbf{p}(t)-\overline{\mathbf{p}}(t)\| \leq \frac{\varepsilon(1+\log M)}{a}<\varepsilon
$$

holds for any initial conditions $\mathbf{p}(0)$ and $\overline{\mathbf{p}}(0)$, and for any structure of perturbed intensity matrix.

If the perturbed intensity matrix has a certain structure (e.g., 20 holds), then, in addition, the perturbation bound

$$
\limsup _{t \rightarrow \infty}|E(t, j)-\bar{E}(t, j)|<\frac{10^{3} \varepsilon(7 j+15)}{1-3 \varepsilon}
$$

holds for any $j$.

Setting $d_{n}=4^{n-1}$, applying this approach and using the obtained bounds, we can find the approximation to the limiting mean of the queue, and the limiting probability of the empty queue with truncation error $10^{-5}$ for $n=22$ and $t \in[23,23.1]$ (see Figs. 21, 24).

Example 7. Let the intensities have the form

$$
\begin{aligned}
\lambda_{n}(t) & =\lambda(t)=10^{3}(1+\sin 0.2 \pi t), \\
\mu_{n}(t) & =\min (n, S) \mu(t) \\
& =\min (n, 1000)(1-\sin 0.2 \pi t), \\
\beta(t) & =2+\cos 0.2 \pi t, \\
r_{n}(t) & =\frac{1+\sin 0.2 \pi t}{4^{n}} .
\end{aligned}
$$

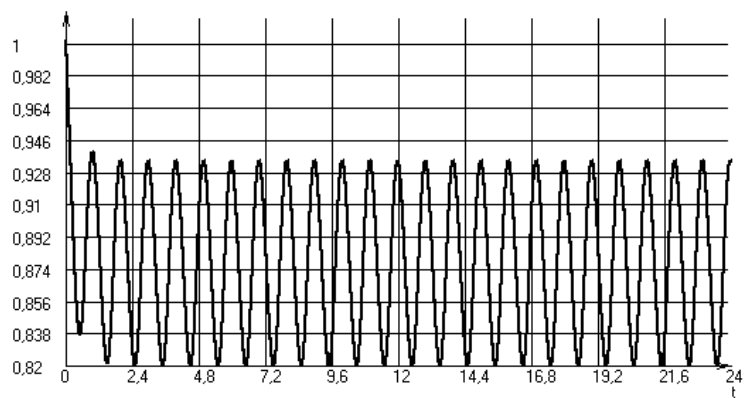

Fig. 20. Example 5: approximation of the probability of the empty queue $\operatorname{Pr}\{\bar{X}(t)=0 \mid \bar{X}(0)=0\}$ on $[0,24]$.
Then the process $X(t)$ is weakly ergodic, has the limiting 10 -periodic regime, and the bound

$$
\left\|\mathbf{p}^{*}(t)-\mathbf{p}^{* *}(t)\right\| \leq 2 \mathrm{e}^{-\int_{0}^{t} \beta(\tau) \mathrm{d} \tau} \leq 10 \mathrm{e}^{-2 t}
$$

holds for any initial conditions $\mathbf{p}^{*}(0), \mathbf{p}^{* *}(0)$ and any $t \geq$ 0 . Moreover, the inequality (12) is fulfilled for $k=2 \cdot 10^{3}$, $\rho=0.5$; hence $X(t)$ has the limiting (10-periodic) mean and the bound

$$
|E(t, j)-E(t, 0)| \leq 5 \cdot 10^{3}(j+1) \mathrm{e}^{-t}
$$

holds for any initial condition $j$ and any $t \geq 0$.

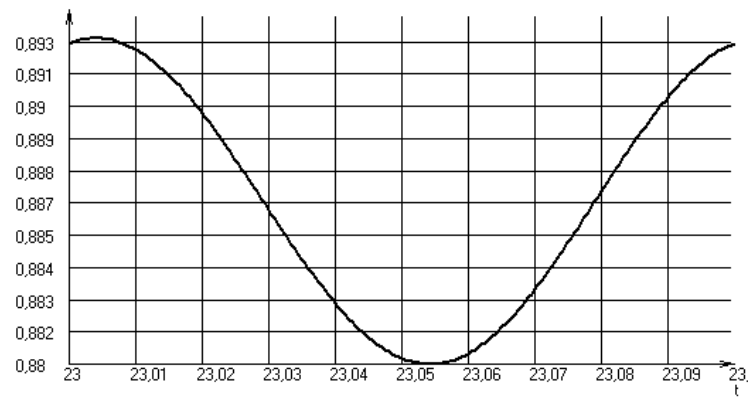

Fig. 21. Example 6: approximation of the limiting probability of the empty queue $\operatorname{Pr}\{\bar{X}(t)=0 \mid \bar{X}(0)=0\}$ on $[23,23.1]$.

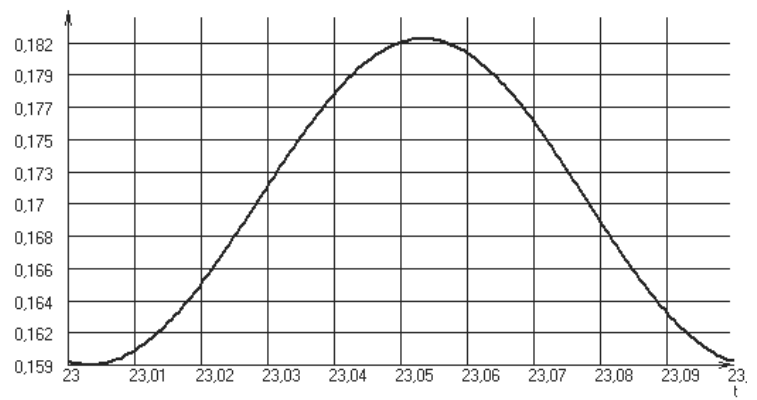

Fig. 22. Example 6: approximation of the limiting mean $E(t, 0)$ on $[23,23.1]$.

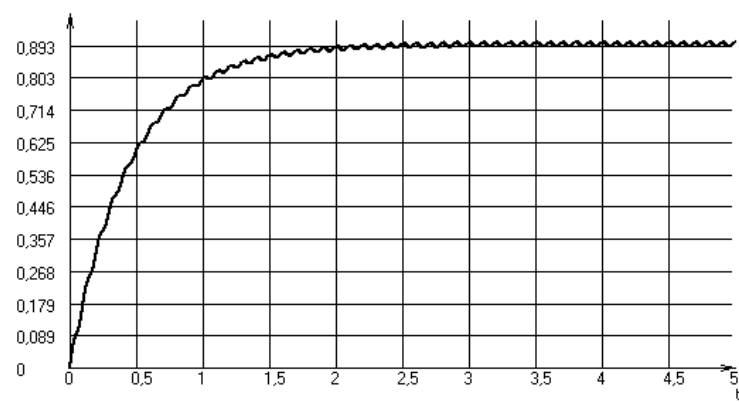

Fig. 23. Example 6: approximation of the probability of the empty queue $\operatorname{Pr}\{\bar{X}(t)=0 / \bar{X}(0)=22\}$ on $[0,5]$. 
Now we have $M=\mathrm{e}^{10 / \pi}$ and $a=2$; hence, the respective perturbation bound

$$
\limsup _{t \rightarrow \infty}\|\mathbf{p}(t)-\overline{\mathbf{p}}(t)\| \leq \frac{\varepsilon(1+\log M)}{a}<6 \varepsilon
$$

holds for any initial conditions $\mathbf{p}(0)$ and $\overline{\mathbf{p}}(0)$, and for any structure of perturbed intensity matrix.

If the perturbed intensity matrix has a certain structure (e.g., 201 holds), then, in addition, the perturbation bound

$$
\limsup _{t \rightarrow \infty}|E(t, j)-\bar{E}(t, j)|<\frac{10^{3} \varepsilon(15 j+70)}{1-3 \varepsilon}
$$

holds for any $j$.

Setting $d_{n}=4^{n-1}$, applying this approach and using the obtained bounds, we can find an approximation to the limiting mean of the queue, and the limiting probability of the empty queue with a truncation error of $10^{-5}$ for $n=22$ and $t \in[23,33]$ (see Figs. 25, 28).

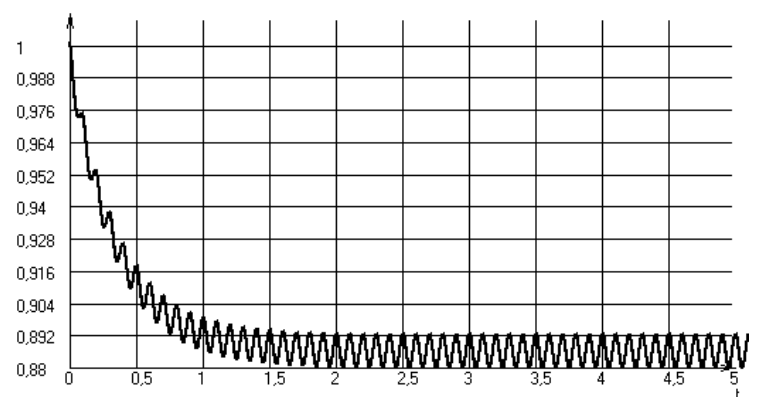

Fig. 24. Example 6: approximation of the probability of the empty queue $\operatorname{Pr}\{\bar{X}(t)=0 \mid \bar{X}(0)=0\}$ on $[0,5]$.

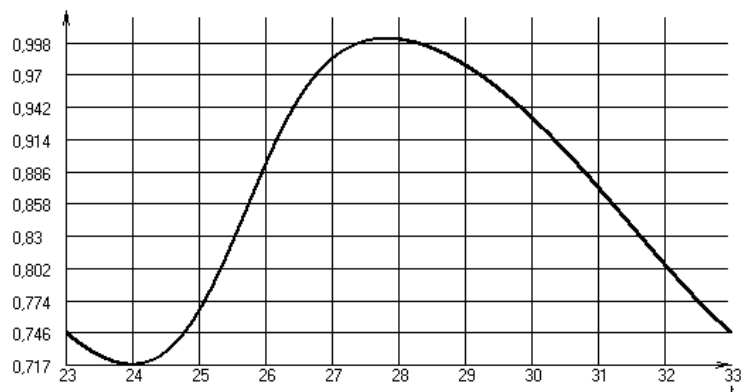

Fig. 25. Example 7: approximation of the limiting probability of the empty queue $\operatorname{Pr}\{\bar{X}(t)=0 \mid \bar{X}(0)=0\}$ on $[23,33]$.
Example 8. Let the intensities have the form

$$
\begin{aligned}
\lambda_{n}(t) & =\lambda(t)=10^{3}(1+0.5 \sin 2 \pi t+0.5 \sin 20 \pi) \\
\mu_{n}(t) & =\min (n, S) \mu(t) \\
& =\min (n, 1000) \\
& =(1-0.5 \sin 2 \pi t-0.5 \sin 20 \pi t) \\
\beta(t) & =2+0.5 \cos 2 \pi t+0.5 \cos 20 \pi \\
r_{n}(t) & =\frac{1+0.5 \sin 2 \pi t+0.5 \sin 20 \pi t}{4^{n}}
\end{aligned}
$$

Then the process $X(t)$ is weakly ergodic, has the

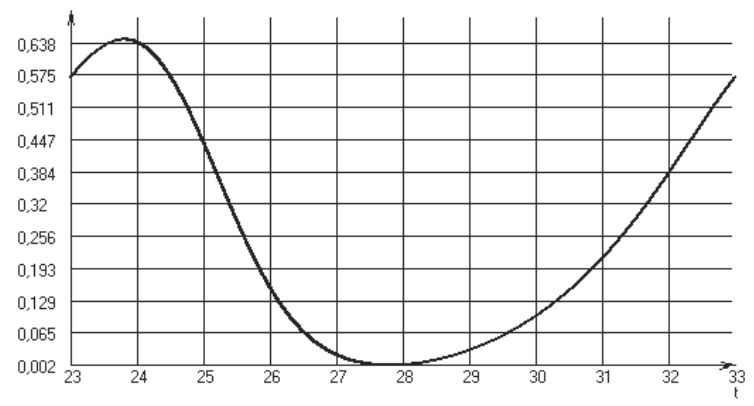

Fig. 26. Example 7: approximation of the limiting mean $E(t, 0)$ on $[23,33]$.

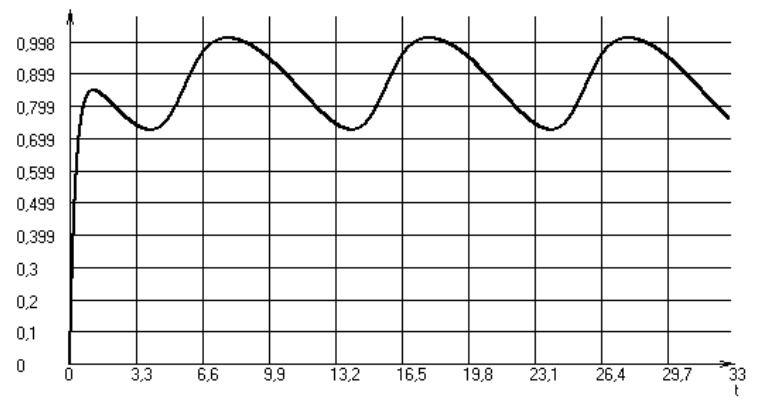

Fig. 27. Example 7: approximation of the probability of the empty queue $\operatorname{Pr}\{\bar{X}(t)=0 / \bar{X}(0)=22\}$ on $[0,33]$.

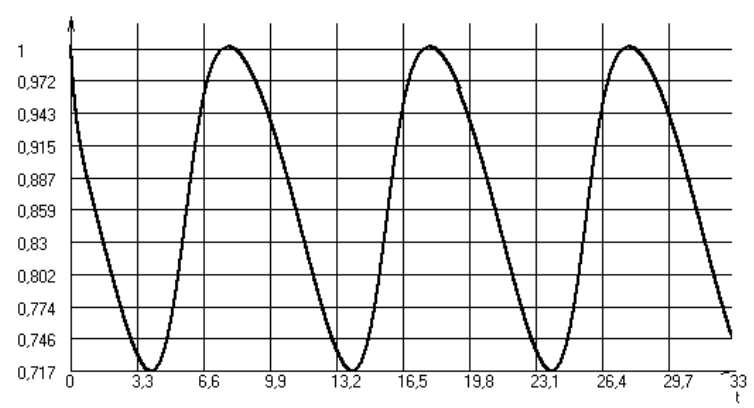

Fig. 28. Example 7: approximation of the probability of the empty queue $\operatorname{Pr}\{\bar{X}(t)=0 \mid \bar{X}(0)=0\}$ on $[0,33]$. 
limiting 1-periodic regime, and the bound

$$
\left\|\mathbf{p}^{*}(t)-\mathbf{p}^{* *}(t)\right\| \leq 2 \mathrm{e}^{-\int_{0}^{t} \beta(\tau) \mathrm{d} \tau} \leq 4 \mathrm{e}^{-2 t}
$$

holds for any initial conditions $\mathbf{p}^{*}(0), \mathbf{p}^{* *}(0)$ and any $t \geq$ 0 . Moreover, the inequality (12) holds for $k=2 \cdot 10^{3}$, $\rho=0.5$; hence, $X(t)$ has the limiting (1-periodic) mean and the bound

$$
|E(t, j)-E(t, 0)| \leq 3 \cdot 10^{3}(j+1) \mathrm{e}^{-t}
$$

holds for any initial condition $j$ and any $t \geq 0$. We now have $M=e^{1 / 2 \pi+1 / 20 \pi}$ and $a=2$; hence the corresponding perturbation bound

$$
\limsup _{t \rightarrow \infty}\|\mathbf{p}(t)-\overline{\mathbf{p}}(t)\| \leq \frac{\varepsilon(1+\log M)}{a}<\varepsilon
$$

holds for any initial conditions $\mathbf{p}(0)$ and $\overline{\mathbf{p}}(0)$, and for any structure of the perturbed intensity matrix.

If the perturbed intensity matrix has a certain structure (e.g., (20) holds), then, in addition, the perturbation bound

$$
\limsup _{t \rightarrow \infty}|E(t, j)-\bar{E}(t, j)|<\frac{10^{3} \varepsilon(8 j+16)}{1-3 \varepsilon}
$$

holds for any $j$.

Setting $d_{n}=4^{n-1}$, applying this approach and using the obtained bounds, we can find an approximation to the limiting mean of the queue, and the limiting probability of the empty queue with a truncation error of $10^{-5}$ for $n=22$ and $t \in[23,24]$ (see Figs. 29, 32).

\section{Conclusion}

Time-dependent analysis of an inhomogeneous birth and death process with an additional arrival from/to origin was carried out. All possible transition intensities were assumed to be non-random functions of time. To the

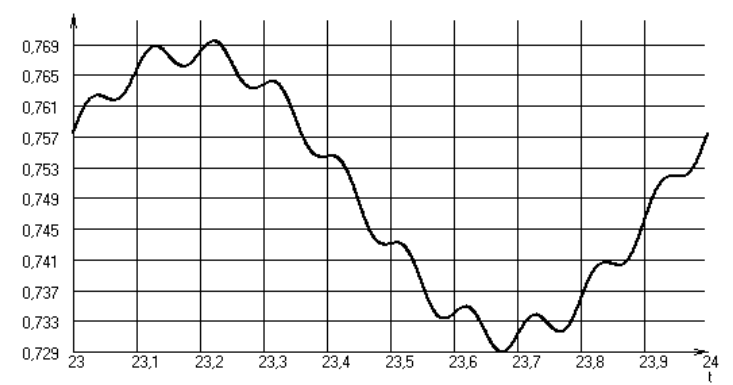

Fig. 29. Example 8: approximation of the limiting probability of the empty queue $\operatorname{Pr}\{\bar{X}(t)=0 \mid \bar{X}(0)=0\}$ on $[23,24]$. best of our knowledge, first ergodicity and perturbation bounds for such a model were obtained, which allow us to find limiting performance characteristics for an arbitrary finite number of servers with a given error. All obtained results were compared with simulation and showed good accuracy. It is worth noting that obtaining results for time-dependent characteristics may be a time consuming way because the number of replications that leads to accurate results requires a careful investigation. Nevertheless, the use of simulation in further studies of possible optimization problems is a promising research direction.

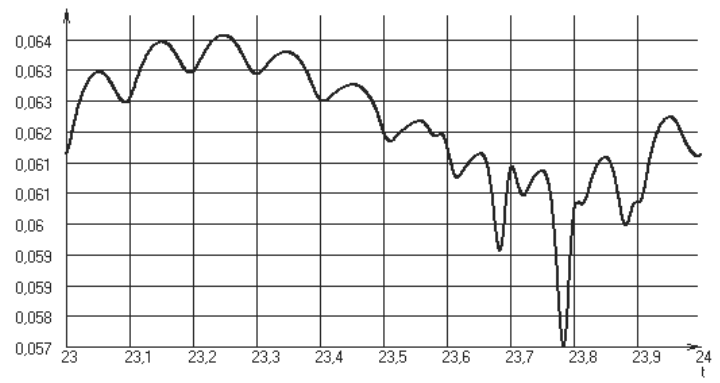

Fig. 30. Example 8: approximation of the limiting mean $E(t, 0)$ on $[23,24]$.

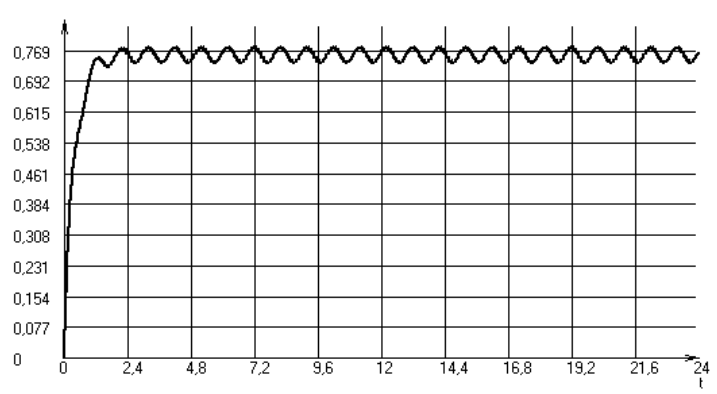

Fig. 31. Example 8: approximation of the probability of the empty queue $\operatorname{Pr}\{\bar{X}(t)=0 / \bar{X}(0)=22\}$ on $[0,24]$.

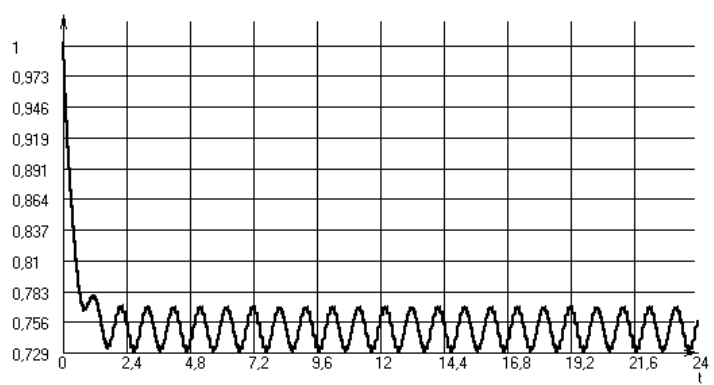

Fig. 32. Example 8: approximation of the probability of the empty queue $\operatorname{Pr}\{\bar{X}(t)=0 \mid \bar{X}(0)=0\}$ on $[0,24]$. 


\section{Acknowledgment}

This work was supported by the Russian Scientific Foundation (Grant No. 14-11-00397).

\section{References}

Chen, A., Pollett, P., Li, J. and Zhang, H. (2010). Markovian bulk-arrival and bulk-service queues with state-dependent control, Queueing Systems 64(3): 267-304.

Chen, A. and Renshaw, E. (1997). The $m|m| 1$ queue with mass exodus and mass arrives when empty, Journal of Applied Probability 34(1): 192-207.

Chen, A. and Renshaw, E. (2004). Markov bulk-arriving queues with state-dependent control at idle time, Advances in Applied Probability 36(2): 499-524.

Daleckij, J. and Krein, M. (1975). Stability of solutions of differential equations in Banach space, Bulletin of the American Mathematical Society 81(6): 1024-102.

Gaidamaka, Y., Pechinkin, A., Razumchik, R., Samouylov, K. and Sopin, E. (2014). Analysis of an $M / G / 1 / R$ queue with batch arrivals and two hysteretic overload control policies, International Journal of Applied Mathematics and Computer Science 24(3): 519-534, DOI: 10.2478/amcs-2014-0038.

Granovsky, B. and Zeifman, A. (2004). Nonstationary queues: Estimation of the rate of convergence, Queueing Systems 46(3-4): 363-388.

Li, J. and Chen, A. (2013). The decay parameter and invariant measures for Markovian bulk-arrival queues with control at idle time, Methodology and Computing in Applied Probability 15(2): 467-484.

Parthasarathy, P. and Kumar, B.K. (1991). Density-dependent birth and death processes with state-dependent immigration, Mathematical and Computer Modelling 15(1): 11-16.

Van Doorn, E., Zeifman, A. and Panfilova, T. (2010). Bounds and asymptotics for the rate of convergence of birth-death processes, Theory of Probability and Its Applications 54(1): 97-113.

Zeifman, A. (1995). Upper and lower bounds on the rate of convergence for nonhomogeneous birth and death processes, Stochastic Processes and Their Applications 59(1): 157-173.

Zeifman, A., Bening, V. and Sokolov, I. (2008). ContinuousTime Markov Chains and Models, Elex-KM, Moscow.

Zeifman, A. and Korolev, V. (2014). On perturbation bounds for continuous-time Markov chains, Statistics \& Probability Letters 88(1): 66-72.

Zeifman, A. and Korotysheva, A. (2012). Perturbation bounds for $M_{t} / M_{t} / N$ queue with catastrophes, Stochastic Models 28(1): 49-62.
Zeifman, A., Korotysheva, A., Satin, Y. and Shorgin, S. (2010) On stability for nonstationary queueing systems with catastrophes, Informatics and Its Applications 4(3): 9-15.

Zeifman, A., Leorato, S., Orsingher, E., Satin, Y. and Shilova, G. (2006). Some universal limits for nonhomogeneous birth and death processes, Queueing Systems 52(2): 139-151.

Zeifman, A., Satin, Y., Korolev, V. and Shorgin, S. (2014). On truncations for weakly ergodic inhomogeneous birth and death processes, International Journal of Applied Mathematics and Computer Science 24(3): 503-518, DOI: 10.2478/amcs-2014-0037.

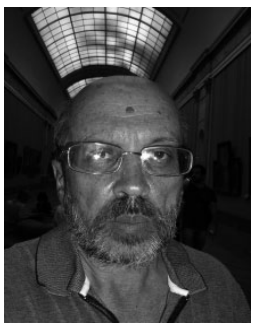

Alexander Zeifman is a Doctor of Science in physics and mathematics, a professor, the head of the Department of Applied Mathematics at Vologda State University, a senior scientist at the Institute of Informatics Problems of the Federal Research Center in Computer Science and Control of the Russian Academy of Sciences, and a principal scientist at the Institute of SocioEconomic Development of Territories of the Russian Academy of Sciences. His current research activities focus on inhomogeneous Markov chains and queueing theory.

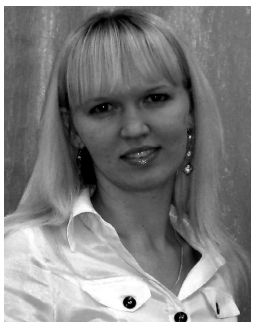

Anna Korotysheva is a Candidate of Science (Ph.D.) in physics and mathematics and a senior lecturer at Vologda State University. Her current research activities focus on queueing theory.

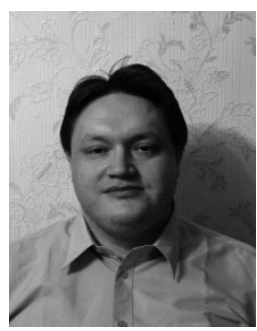

Yacov Satin is a Candidate of Science (Ph.D.) in physics and mathematics, and an associate professor at Vologda State University. His current research activities focus on inhomogeneous Markov chains and queueing theory.

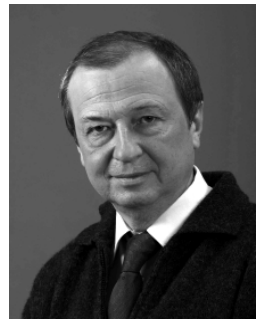

Victor Korolev is a Doctor of Science in physics and mathematics, a professor, the head of the Department of Mathematical Statistics at the Faculty of Computational Mathematics and Cybernetics of M.V. Lomonosov Moscow State University, and the leading scientist at the Institute of Informatics Problems of the Federal Research Center in Computer Science and Control of the Russian Academy of Sciences. His current research activities focus on probability as well as statistic theory and models. 


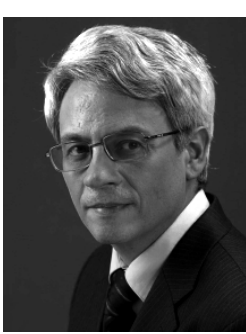

Sergey Shorgin is a Doctor of Science in physics and mathematics, a professor and the deputy director of the Institute of Informatics Problems at the Federal Research Center in Computer Science and Control of the Russian Academy of Sciences. His current research activities focus on probability and statistics (theory and models).

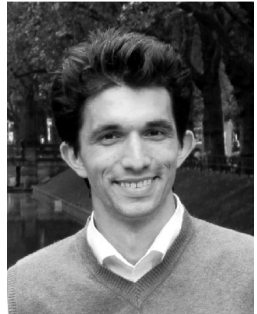

Rostislav V. Razumchik received his Ph.D. in physics and mathematics in 2011. Since then, he has worked as a senior researcher at the Institute of Informatics Problems of the Federal Research Center in Computer Science and Control of the Russian Academy of Sciences, and as an assistant professor at Peoples' Friendship University of Russia. His current research activities focus on queueing theory and simulation.

Received: 28 November 2014

Revised: 20 January 2015

Re-revised: 7 August 2015 